\title{
General Analysis on the Negative Emotions over the Inherited Work of Twelve Muqam Uygur Xinjiang, China*
}

\author{
Adila Aimaiti \\ Institute of Uighur Language and Culture \\ Northwest Minzu University \\ Lanzhou, China 730001 \\ Kashgar Art School \\ Kashgar, China 844001
}

\author{
Nuermaimaiti Yimamu \\ Institute of Uighur Language and Culture \\ Northwest Minzu University \\ Lanzhou, China 730001 \\ Kashgar Art School \\ Kashgar, China 844001
}

\begin{abstract}
Under the attention over the work of folk culture's inheritance and development by the communist party and government, we have already been entitled with the superior conditions to learn and inherit the "Twelve Muqam" Uygur China. However with the development of economy and the acceleration of urbanization process, the teenagers and young generation are becoming less and less enthusiastic about studying Uygur Twelve Muqam". This article will state the real situation about the 1st singing competition of "Twelve Muqam", the attitude of the successor of "Twelve Muqam" over this competition from Xinjang Uygur, as well as the current and the future direction of the inherited work of muqam art.
\end{abstract}

\section{Keywords-Twelve Muqam; inherit; chong naghmah}

\section{INTRODUCTION}

As is known to all, under the work of saving and discovering folk culture by the communist party and the government, and based on the further understanding of "Twelve Muqam", this bright folk art, our country and people have adopted various ways to bring back the glory of the "Twelve Muqam" which is on the edge of extinction caused by its historical reasons. During year 1950-1955, Turdi Axun and other masters of Muqam was invited twice to Urumqi and recorded the whole album of "Twelve Muqam". 1 On the 6th of August 1956, Turdi Axun passed away. Before saying goodbye to this world, he successfully passed these precious cultural heritage accumulated generation by generation to the next generation. Several

*Funded project: year 2017 national social science fund of art department youth project - stage research outcome of "Intangible cultural heritage of Twelve Muqam academy development" (approval no. 17CH219); year 2015 national social science western project - stage research outcome of "A special study of Uygur folk oral Poetics" (approval no. 15BZW196 ); year 2018 Northwest National University Central College essential science research fund for post-graduate project - stage research outcome of "The development and inheritance mechanism of Twelve Muqam Uyghur" (approval no. Yxm2018004).

Yusman Crangi. Historic process of recording, selecting, writing and publishing Ikki Muqam art[J]. Quan, 2016, (5), 88. years later, all left are some scattered broken words recorded by History of Musicians, or some parts from "Dastan" or "Mashrap", however "Naghma" part will never been seen in "Twelve Muqam".

In 1960, "Twelve Muqam" was completed based on the recorded edition done by the Muqam masters, and the stave book was first published by People's Music Publishing House and The Ethnic Publishing House. In 1964 the original lyric book of "Twelve Muqam" provided by Turdi Axun was issued internally. 2 In 1993, the twelve set of "Twelve Muqam", text book and recorded tape of the twelve set music score by Xinjiang Muqam Art Group was issued. In 1997, after being complemented and sorted three times, the 13 books and text books were published. In 2002, based on the 3rd edition, CD and DCD records were released, which has provided strong and solid hardware for students of "Twelve Muqam". In order to guarantee the complete inheritance of "Twelve Muqam" art and enhance the inherited efficiency, from year 1996, Xinjiang Arts University has opened the professional Muqam performance class, which is dedicated to make a historic change on the saving, protection, research and development of "Twelve Muqam". 3 Based on the books and recorded tapes issued in 1993, art troupes from all places, cities and counties, promote the scale of learning Muqam, and has formed a status of having at least one or two Muqam singer who are capable to sing one or two Mu Card Mar or Dastan's melody. Xinjiang Arts University has trained 9 groups of Muqam's successors altogether 180 people, which is a huge contrast compared to 5 Muqam artists back then during the past 50 years. Although under the boost of above-mentioned working process, we are qualified with the relatively superior conditions of learning and inheriting "Twelve Muqam", young generations are becoming less and less enthusiastic in

\footnotetext{
Yusman Crangi. Historic process of recording, selecting, writing and publishing Ikki Muqam art[J]. Quan, 2016, (5), 88.

Ji Zhou, Imin Akomat. Analysis on Uygur music treasureMuqam: oral and intangible heritage representative work[M]. Urumqi: Xinjiang Science and Technology Press, 2007:226.
} 
learning "Twelve Muqam" due to the speeding up of economic development and urbanization process.

Currently, unbalanced geographic phenomenon appears in the inherited work of "Twelve Muqam", which results in certain negative emotions, especially the "Naghma" part is not known to public. In the crowds, particularly young and mid-aged Muqam successors are not widely accepted. And the reasons are analyzed below.

\section{THE REASON WHY "NAGHMA" OF TWELVE MUQAM Is Not POPUlAR AMONG THE PEOPLE}

Firstly, the "Naghma" part of "Twelve Muqam" is the key part, holding the heart position. The structure of "Twelve Muqam" is divided into 4 parts: $\mathrm{Mu}$ Card Mar (part of Muqam), Naghma or Dastan and Mashrap.4From the whole structure, "Naghma" has only $1 / 4$ of Muqam, but actually, parts of its melody and rhythm structures are very complicated. From the complex of its adjustment and tone's structure sort of speaking, "Naghma" should hold $2 / 3$ of a whole set of Muqam. The rest are the 1/3. "Naghma" is more difficult than "Dastan" and "Mashrap", as well as from the perspective of aesthetics, the performers and the majority of the public are both required a relatively high level in order to better appreciate the essence of Naghma", which is an obstacle for Muqam artists to learn and inherit Muqam. Therefore partial tracks of "Naghma" are no longer performed in Muqam's show.

Secondly, partial configuration of lyrics of "Naghma" is very different from those of "Dastan" and "Mashrap". The main configuration of lyrics of "Naghma" is coming from the key points of classic philosophical metaphysical poetry. Those poems are mixed with ancient Uygur, Chahetai, Persian, Arabic multi-language and multi-cultural layers. In my opinion, a person needs to know at least one of two mentioned languages and their culture to be able to sing "Naghma" perfectly. From the current situation of Muqam artists, we can tell they haven't been exposed to so many language and cultural layers. This is because the students from Muqam professional class of Xinjiang Arts University haven't been arranged to learn such language courses. 5 Thus the graduated Muqam's successors don't know these languages. All cases of professionally trained Muqam's successors are the same, whilst the rest folk Muqam's successors, artists from art \& theatre group and Muqam art staff have even less possibilities to learn these languages. Therefore, Muqam singers are not willing to sing such songs with the classic lyrics. Additionally, this put the public into a difficult level to understand the meaning of the songs, and of course in the end they feel bored and less interesting. Overall speaking, such Muqam is basically impossible to perform the way it should be.

The lyrics of "Dastan" and "Mashrap" are narrative poetry and folk songs, which are mainly used to praise

Yusman Crangi. Discussion on the age of formation of Ikki Muqam[J]. Xinjiang Social Science Forum, 2007, (1).

The author's transcript of a discussion with students from Muqam major Xinjiang Arts University. excellent task examples from the folk. These folk narrative poetry and folk songs are passed on for hundreds of thousands years, with the non-stop innovation and down-toearth context, which make it easy for singers to understand and perform. In the end, such simple rhythm and easy melodies have become more and more popular. Even the public like such melodies because it's easy to accept, appreciate and study.

Appreciation of performance art is very important; moreover the relationship between the performer and the appreciator is also quite critical. Acceptance of aesthetic psychology in aesthetic theory, annoyance, frustration, sense of urgency... and other factors constantly push forward the creation of new concept for the performers. 6 The Naghma's part of "Twelve Muqam" not accepted by the appreciators and not performed by the singers also conforms to the pattern of performance art.

Thirdly, when the economy boosts to develop and acceleration of urbanization, lots of convenience is brought to our life. However, this phenomenon also has many side effects on the Uygur traditional Chinese art. Uygur Twelve Muqam is facing challenges in the culture market, even its inheritance and existence is in crisis, especially the hit on the Naghma's part.

The market produces consumer goods based on the demand of the consumers. Therefore the published videotape of "Twelve Muqam" only selected partial tracks of "Dastan" and "Mashrap", Jura and Sanam songs from Naghma, all the rest are not included. That's because this is decided by the aesthetic demand of our consumers. Moreover, in the annual art performance of all kinds, we hardly can see the performance of "Naghma", so the chance of seeing it is very rare. In wedding and banquet, instead of performing "Naghma", folk songs and live music are performed. In countryside various Mashrap and get-together parties also don't sing "Naghma" but folk songs and partial tracks of "Dastan". Luckily, "Naghma" can still be seen in cities like Kashgar. In parties and gatherings singing and performing partial segments and intermezzo of "Dastan" has formed a routine, but for "Naghma" is really rare (Kashgar region is basically like this, other areas are more or less the same). All in all, the reasons causing the current situation of "Naghma" are the above-mentioned 3 points.

\section{THE REASON WHY "NAGHMA" OF "TWELVE MUQAM" CAUSE NEGATIVE EMOTION IN THE FOLK}

"Twelve Muqam" such big scaled classic work is maintained and protected pretty perfectly. "Twelve Muqam" altogether published three times music scores and lyric books, and two times tapes and one time CD, DCD records, which has formed a solid foundation for the preservation of "Twelve Muqam". In Europe, world-famous composers like Bach, Mozart, and Beethoven, whose music works has been recorded and carried forward till today. From this perspective, "Twelve Muqam" had been recorded and

Tulson Maimaimaiti Shawuti, Imin Akomat. Base of Art theory [M]. Urumqi: Xinjiang People Publishing House September 2005, 299. 
conserved since 1950s, which is considered as a great achievement. In this technology boosting modern society, we no longer need to worry about preserving the culture. Nevertheless we need to pay special attention to one point that "Twelve Muqam" is a complexed music, it includes not only music but also the song's part. All the materials we have recorded are spiritless, no matter how advanced the technology is and how precisely the music stave records its music, live songs can never been recorded and revealed its true colors in such manner. Moreover the recorded music maybe never could be returned to the original "Muqam" due to the variation of art talent, level of performance and the way of expression. So far, in our country a question about the inherited work on the unofficial civilization heritage is being ignored - recorded edition is used to replace the live performance, which has created wrong opinions over the way of inheritance. We need to remember these two things must not be confused. The dead written edition can never replace the edition of live oral performance. This opinion will impact the education of Muqam's inherited work in a historic way. I propose working on learning "Naghma" to be an important part during the inherited work of Twelve Muqam, otherwise following such trend, partial melodies of "Naghma" (especially for the rhythm structure complicated melodies like Taieza, Nusha, Khikserika) will soon disappear from the nation or slowly has become music without songs. We just said before, partial melodies and lyrics of "Naghma" are configured with classic poetries and written in many languages, which make ordinary people very hard to understand, thus the crowds won't show interests. The disappearing lyrics are a proof of showing such music will be transformed to music without songs and lyrics. We need to remember such extinction appeared once in the history, which is the transformation of Ili. The above-mentioned opinions are just hypothesis over the negative consequences that might possibly appear.

In the era of rapid economic development and accelerated urbanization, media is acting as a catalyst in training and educating the successors of Twelve Muqam. They broadcast Twelve Muqam to the crowds. The more people see, the more mature people's aesthetics become. Therefore, I think Media should fully realize their responsibilities in cultivating Twelve Muqam's successors and preventing "Naghma" from vanishing from "Twelve Muqam".

Certainly, in the market economy era, we cannot make demands on the production of market products but produce products based on the consumers' demands. We cannot put Twelve Muqam such classic art work to market in big quantities and reduce the contemporary art. Because the consuming demand of majorities are contemporary stuff. But we can use media to train people's interests by taking advantages of originally created melodies and songs by Twelve Muqam and put it to the market to bring broad attention of people as well as to increase the enthusiasm of Twelve Muqam's successors.

Songs and music are art forms by using voices to express human being's internal feelings. As the one of the most ancient art forms, human never lives without music, which constantly brings people happiness and plays a positive role in constructing wonderful lives and harmonious society. With the rapid economic development and popularization of social media, foreign songs have been penetrated massively into our lives, which has attracted attentions of art lovers with innovative spirits. As a result, a number of original songs with time spirits have emerged, which certainly influenced in the field of Uygur art meanwhile having concrete audiences and position. However some of our conservative people often judge others with their own thoughts and reject such innovation. Like what we said before, "Muqam" is not liked by some young and mid-young aged people, neither by mid-aged and adults concerning such creative melodies.

Folk songs and Twelve Muqam are actually originalcreated songs by certain artist in a certain epoch. With the time passing by, they were passed on slowly to the people. Through years of being filtered, selected, eliminated, and reprocessed, the name of the author with his work well liked by people has been forgotten gradually. Only the title of "People" has been still conserved till today with such "Folk Songs", "Folk Music" and "Muqam" these names. In fact, these melodies were the most classic originally-created songs back then. The concept of "Creative Songs" is a relative concept, with the time flying, the meaning of this concept is also changing. For example, the now converted folk song "The Spring of Kashgar" by composer Tuldi Mezi, "The Child Says the Moon" by composer Abela Abdullah, etc. "Morality is the Decoration of People" by composer Tulson Cadel, "The Age of Liberation" by composer Yushanjiang Gamey and so on. These songs were actually the classic creative songs but nowadays, many young people have forgotten those creators and taken these songs as "Folk Songs". Maybe decades later the creators of these songs would be forgotten completely, and these songs would be officially listed as "Folk Songs". Therefore, we cannot reject newly created songs and should treat them positively. In consideration of its relationship with Uygur art in the future development process, we should accept it with scientific attitude. Additionally young composers should also accept the merits of Western vocal music art accordingly, by absorbing and taking advantage of the advanced parts, in order to create big amounts of newly creative melodies with national and contemporary style based on the Uygur folk songs and the spirits of Muqam, so as to push forward the development of construction of socialist spiritual civilization and satisfy the needs of daily increased spiritual culture of the people.

\section{CONCLUSION}

All in all, I'm quite confident that some problems ignored in the inherited work of "Twelve Muqam" Uygur Xinjiang China will be resolved comprehensively and mapped out as planned in the future. Successors of Muqam art from all over the places will emerge like bamboo shoots after a spring rain. The priceless treasure, "Twelve Muqam Uygur Xinjiang China" of Chinese culture treasury will rebounce back and forth on the world's stage to make the whole world realize 
the great Chinese culture and the excellent art works of Chinese culture.

\section{REFERENCES}

[1] Ji Zhou. Muqam Music Uygur Xinjiang China[M]. Beijing: Central Conservatory of Music Press, 2008: 184-204 (In Chinese).

[2] Tulson Maimaimaiti Shawuti, Imin Akomat. Base of Art theory[M]. Urumqi: Xinjiang People Publishing House 2005: 273-338 (In Chinese).

[3] Ji Zhou, Imin Akomat. Analysis on Uygur music treasure-Muqam: oral and intangible heritage representative work[M]. Urumqi: Xinjiang Science and Technology Press, 2007:226 (In Chinese).

[4] Abdushikul Mohammed Yiming. Uygur Muqam treasury [M]. Urumqi: Xinjiang People Publishing House, August 2009, 329 (In Chinese).

[5] Yusman Crangi. Discussion on the age of formation of Twelve Muqam[J]. Xinjiang Social Science Forum, 2007, (1) (In Chinese).

[6] Yusman Crangi. Historic process of recording, selecting, writing and publishing Twelve Muqam art[J]. Quan, 2016, (5), 88 (In Chinese).

[7] Ayatiguli Niyaz. Discussion on the two styles of song's creation[J]. Xinjiang Art, 2017, (3), 68 (In Chinese). 